\title{
Map Design in Subway Stations through Passengers' Wayfinding Behavior Perspective
}

\author{
Pei-Ying Li ${ }^{1}$, Meng-Cong Zheng², Haruo Hibino ${ }^{1}$, Shinichi Koyama ${ }^{1}$ \\ ${ }^{1}$ Design Psychology Unit, Department of Design Science, Chiba University, Japan \\ 2 Environment Behavior Lab, Department of Wood Science and Design, \\ National Pingtung University of Science and Technology, Taiwan \\ zoelady@gmail.com
}

\begin{abstract}
This empirical study of behavioural observation with a verbal report attempts to understand the process of map-reading for purposes of way finding in a subway station at Taipei in Taiwan. Subjects were required to find the shortest way in reaching an exit via three manners - without a map, aided by 2D, and 3D map. The results indicated that the design of underground space maps should support a direction and floor wayfinding strategy, plus the selection of a suitable angle of vision.. Important considerations include marking the nearest exit, marking scale, intensifying wall performance, and clarifying the discrimination of different floors and areas.
\end{abstract}

Keywords: subway station, map-reading, map design, wayfinding

eISSN 2514-751X @ 2017 The Authors. Published for AMER ABRA by e-International Publishing House, Ltd., UK.. This is an open access article under the CC BY-NC-ND license (http://creativecommons.org/licenses/by-ncnd/4.0/). Peer-review under responsibility of AMER (Association of Malaysian Environment-Behaviour Researchers), ABRA (Association of Behavioural Researchers on Asians) and cE-Bs (Centre for EnvironmentBehaviour Studies), Faculty of Architecture, Planning \& Surveying, Universiti Teknologi MARA, Malaysia.

https://doi.org/10.21834/aje-bs.v2i2.181 


\subsection{Introduction}

In urban cities, large subway stations are the public space most frequented by the general masses, and thus the issue of how people can move smoothly underground has attracted concern. Subway stations usually include a lot of areas and floors. Therefore, way-finding behavior involves not just 2-D navigation, but also that of 3-D navigation. Recently, many studies have worked on 3-D navigation; as observed by Christoph (2006), in a study of way-finding strategy in multi-level buildings that focused on 12 participants to conducting way-finding study in a building which comprises of eight areas and four floors. Participants had to use direction strategy (the horizontal position of the goal) and floor strategy (for level-changes) to reach assigned floor and area. In subway stations, passengers often change levels and areas to reach specific destinations, such as No.3 exit, or No.2 platform. Consequently, it has demonstrated that analysis of direction and floor strategy for wayfinding at subway stations is necessary.

Subway stations typically display, there are mainly two categories of spatial information, namely directional sign and map to support way-finding. Directional signs support direction information, while map provide survey knowledge. Many studies shows directional signs were used more frequently than maps. The use of maps is thought to have effects upon cognitive maps and way-finding. Rob (2002) indicated the effects of maps. First, training in map use can provide guidance in how to process and comprehend spatial information. Second, studying a map can lead to greater knowledge of an area by revealing real world spatial information derived from direct experience. Third, maps assist way-finders in conducting way-finding planning before way-finding, as well as in predicting environmental context not traveled before. Consequently, way-finders can effectively exploit maps to enhance way-finding accuracy. In addition, maps are considered to be of much help as found in escape behaviour study. Based on simulation experiment results obtained by the NIED (2002) research for subway station shelters, this study finds that the complementary use of directional signs and maps can most effectively support action. Escape maps are commonly installed in subway stations so that passengers can understand the escape route as soon as possible before an accident occurs.

Way-finding processes were generally considered as two aspects of human navigation. The first aspect is route planning, which aims to facilitate target-oriented navigation. The second aspect addresses route modeling and the understanding of how people navigate their environment (David, 2007). Torgny (1987) also indicated that in complex tasks, mapreading in connection with way-finding can be profitably studied in its highly developed forms.

This study investigates the cognitive processes of travelers when navigating subway stations, specifically when those travelers are asked to perform way-finding tasks involving finding their way from a platform to the exit. Behavioral observation and verbal reports are used to examine the processes from map-reading to way-finding performance. Route description is used to get the data of subjects' route knowledge. This work explored spatial 
properties useful to way-finding, and examined the performance of existing maps of the actual environment so as to present suggestions regarding map design for subway stations.

\subsection{Methodology}

\subsection{Survey}

A total of 186 survey questionnaires were returned. All subjects are inhabitants of Taipei who are familiar with Taipei Station. Respondents were asked two main questions: 1) to evaluate Taipei Station based on the Sanja's criteria (2001); and 2) to evaluate sign and map design at Taipei Station. The questions are graded based on a five-point scale: -2 indicates very poor, -1 indicates poor, 0 indicates fair, +1 indicates good, and +2 indicates very good.

\subsection{Investigation}

An empirical investigation related to map-reading and way-finding was conducted with two tasks. Task 1 is to reach an exit without reading a map. Task 2 is to read map, plan a route, memorize the route, describe the route, and find a way to the designated exit. In order to realize this cognitive process, we introduce the verbal reports of way-finders as an additional data source. Christoph (2006) followed Passini (1992) by using the collection of way-finding behavior data to successfully complement the verbal reports of task-concurrent thoughts to obtain a comprehensive picture. Thus, this study is conducted to measure mapreading and way-finding performance, and to analyze protocol reports and verbal route description.

\subsection{Participants}

In the map-reading and way-finding investigation, the 12 study participants comprised graduates of the Education Department of Tung Hai University of Taichung City. All 12 were at that time working as high school teachers. The average age was 29.6 years old. Six of the participants were males, while the other six were females. Six of the participants were unfamiliar with the station, having visited the place fewer than three times, while six were familiar with station, having lived in Taipei City and having used the Taipei MRT to commute for more than 1 year. Three of the participants who were unfamiliar with Taipei City were assigned to read the $2 \mathrm{D}$ map, while the other three were assigned to read the $3 \mathrm{D}$ map.

\subsection{Procedure}

Video was used to record the behavior and protocol reports of individual participants. The experimental site was Taipei Station in Taiwan, a large underground station with three tracks (MRT, Taiwan Railway, and High-speed Rail), and with four underground levels. The 
map sample (Fig.1) used in this study is based on the 2D and 3D maps installed in the MRT station. The 2D map is installed at the centre of the platform, gate, and entrance and exit of every floor. The 3D map is installed in front of and inside the station elevator. To avoid the influence of directional signs on the way-finding task, the exit marker is deleted from the map sample.
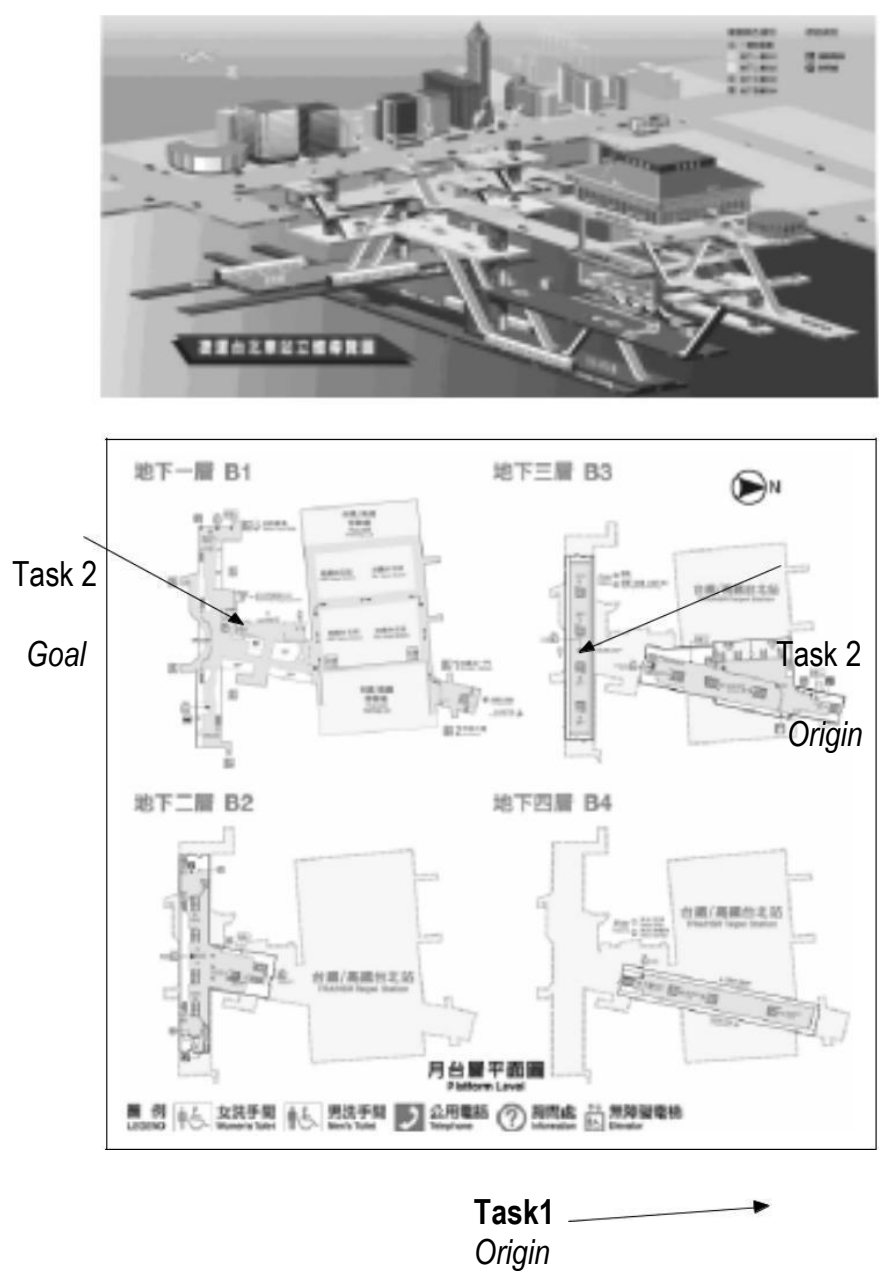

Figure 1: 3D map sample (left) and 2D map sample (right)

(Source: Taipei station, 2008) 
This study examines whether a participant can complete the way-finding task according to the previous way-finding plan under conditions of time pressure and low visibility. Smoke appearing would be the problem encountered in the escape task. Smoke appearing during a disaster can cause blindness, and smoke along escape routes in particular has the potential to cause panic. Participants were thus asked to wear foggy glasses which were made from some opaque material. This reduced visibility to $20-30 \mathrm{~m}$ (about reduction coefficient at 0.1) (Ding-Lin, 2007). This study confirms that each participant can walk safely wearing foggy glasses and can also see directional signs.

\subsubsection{Verbalization}

Verbal protocols for map-reading are classified into three types: smooth, problematic, and route memorization. Verbal protocols for way-finding arej @classified into four types: recognizing route smoothly, problematic, searching for, and recalling route. Besides these, this study performs coding of verbal protocols for directional and floor strategies, based on the definition by Christoph (2006). Thus, each sentence has two codes. After coding, the verbalization was analyzed by an ANOVA test with factors map usage (2D map used, 3D map used) and familiarity (familiar, unfamiliar).

\subsubsection{Route description}

Brosset (2007) indicated that the proposed model incorporates the three main components of a route description, as previously identified by Michon and Denis: namely action, landmark and spatial entities. An action represents the displacement behaviour of a human acting in the environment. A landmark is the most salient feature used in human navigation. Spatial entities denote two-dimensional entities on which moves are executed (e.g. a path) or non-salient and non-punctual entities used in navigation (e.g. an area). In this study, the landmark and spatial entities are those used by Passini's (1992) Elements of underground space. Statistical analysis was performed on the usage frequency of various elements. He defined them in the following way: 1. Paths are the circulation system, which include corridors, promenades that are part of the horizontal circulation and stairs, elevators and escalators are part of the vertical circulation; 2 . Walls inside the building could be viewed as the edges; 3 . Districts are considered to be certain areas with specific functional characteristics; 4. Nodes are defined as important circulation intersections, like halls and indoor squares; 5 . Landmarks are clearly remembered elements such as particular shops, sculptures or decorative elements. According to Passini, not only objects but also the space itself, can serve as reference points, and can be considered as landmarks.

\subsection{Results}

\subsection{Questionnaire Results}


A total of 200 questionnaires were distributed. 186 valid questionnaires were returned. These included 97 from female respondents and 89 from male respondents. The respondents included 21 commuters, 87 individuals who visited the station over 20 times each year, and 78 individuals who visited the station between 5-20 times each year.

For the evaluation of underground stations in Taipei, the average values of six indicator items are as follows: overview, escape, visibility, management and order, way-finding, attractiveness, physiological comfort, and daylight. The results are shown in Table 1. The average evaluation for attractiveness, way-finding and escape is more positive than for the other 3 items. The average evaluation for directional sign is positive $(+0.02)$. However, the average evaluation for map is negative $(-0.08)$. Respondent satisfaction with using maps receives a negative evaluation, indicating the need to improve map design.

Table 1: The averages values of six indicator items

\begin{tabular}{cccccccc}
\hline overview & escape & spatial structure & safety & wayfinding & attractive & comfortable & daylight \\
-0.31 & -0.42 & -0.29 & -0.26 & -0.63 & -0.65 & -0.3 & -0.18 \\
\hline
\end{tabular}

\subsection{Results Of Investigation}

\section{Task 1}

In this task, all participants were asked to find their way to any ground exit from the Danshui line platform in the B4 floor without reading a map. Five participants arrive at the exit of the Danshui line, and seven arrive at the exit of Banqiao-Nangang line. Only two subjects reached the nearest exit, but no subject took the shortest path to the nearest exit. All subjects reached the exit successfully, and their performance is shown in Table 2. Higher stop frequency was observed for subjects unfamiliar with the site $(p<0.016)$, as well as longer stop periods $(p<0.029)$. Additionally, respondents unfamiliar with the site reported greater problems than those familiar with the site $(p<0.03)$. Furthermore, the covered distance was $53 \%$ longer than in the shortest possible route. Subjects familiar with Taipei Station moved more smoothly than unfamiliar subjects according to verbalization of problems encountered. Familiar subjects did not perform better in terms of walking distance and duration than unfamiliar subjects. Task 1 asked participants not to read a map while performing their way-finding. The results indicate that no one took the shortest route to the nearest exit, and over half of subjects failed to find the nearest exit at all. Thus, it is not easy for subjects to find the shortest route to the nearest ground exit by showing them directional signs only. 
Table 2: The average of performance, behaviour measures, and verbalization

\begin{tabular}{lccccc|cc}
\hline \multicolumn{9}{c}{ Performance } & \multicolumn{2}{c}{ Verbalization } \\
& Time & Distance & PAO & Stops & Stop & smooth & problematic \\
& $(\mathbf{s e c})$ & $(\mathbf{m})$ & & $(\mathbf{n})$ & time $(\mathbf{s e c})$ & $(\mathbf{n})$ & $(\mathbf{n})$ \\
Unfamiliar & 266 & 214.85 & 1.46 & 1.83 & 17.17 & 10.17 & 2.33 \\
Familiar & 286 & 252.13 & 1.60 & 0.75 & 14.37 & 11.67 & 0.17 \\
& & & & & & & \\
\hline
\end{tabular}

\section{Task 2}

\section{Results of the performance measures}

Task 2 involves way-finding from the platform of the Banqiao-Nangang line to exit M4. Half of the subjects select the same route, but it is not the shortest route. Those subjects selected the nearest stairway in front at first.

The results are shown in Table 3. Subjects using 2D and 3D maps exhibited significant differences in terms of loss. ( $p<0.001)$ Five subjects who read $2 \mathrm{D}$ maps completed the way-finding task once. Seven subjects got lost or reached the wrong exit (six 3D map subjects, and one 2D map subject). Unfamiliar subjects spent over three times longer on map reading than familiar subjects, and thus required more time to complete the task ( $p<$ 0.001 ). All of the $3 \mathrm{D}$ map subjects completed the task twice, and the total way-finding time for the 3D map subjects exceeded that of the 2D map subjects $(p<0.023)$. The way-finding and detour distance are also longer for 3D map subjects compared to those of $2 \mathrm{D}$ map subjects $(p<0.038)$.

Table 3: Results of route planning and way-finding for subjects map usage and familiarity

\begin{tabular}{|c|c|c|c|c|c|c|}
\hline & & \multicolumn{2}{|c|}{ Map usage } & \multicolumn{2}{|c|}{ Familiarity } & \multirow[t]{2}{*}{ Total } \\
\hline & & $2 \mathrm{D}$ & $3 D$ & unfamiliar & familiar & \\
\hline \multirow[t]{3}{*}{ Map-reading } & $\begin{array}{l}\text { Planning route } \\
\text { distance }(\mathrm{m})\end{array}$ & 184.2 & 189.86 & 185.84 & 188.22 & 187.03 \\
\hline & Planning time (sec) & 175.83 & 234 & 298.5 & 111.33 & 204.92 \\
\hline & $\begin{array}{l}\text { Planning distance/ } \\
\text { the shortest distance }\end{array}$ & 0.32 & 0.36 & 0.32 & 0.33 & 0.32 \\
\hline \multirow[t]{7}{*}{ Way-finding } & $\begin{array}{l}\text { Way-finding } \\
\text { distance }(m)\end{array}$ & 221.04 & 336.87 & 297.59 & 249.87 & 278.96 \\
\hline & Detour $(m)$ & 34.34 & 134.06 & 109.25 & 59.15 & 84.2 \\
\hline & Way-finding time (sec) & 275.17 & 445.33 & 405.17 & 315.3 & 360.25 \\
\hline & Stops $(n)$ & 3.67 & 4.17 & 4.00 & 3.80 & 3.92 \\
\hline & Stop time (sec) & 27.00 & 52.17 & 53.17 & 26.00 & 39.58 \\
\hline & getting lost (n) & 0.17 & 1.00 & 0.67 & 0.50 & 0.58 \\
\hline & Total task time (sec) & 465.5 & 798.67 & 703.67 & 426.67 & 632.08 \\
\hline
\end{tabular}




\section{Results of subjects' verbalizations}

The results of verbalizations are shown in Table 4.The verbalizations of planning problem for $2 \mathrm{D}$ map subjects are lower than those for 3D map subjects $(p<0.37)$. The $2 \mathrm{D}$ and $3 \mathrm{D}$ map subjects differ significantly in terms of direction and floor strategy $(p<0.002)$. The frequency of verbalization regarding direction and floor strategy is almost identical for $2 \mathrm{D}$ map subjects. However, the frequency of floor strategy is half of that of direction strategy for 3D map subjects (Table 4). It shows that 3D readers mostly have problems with direction planning, corridor, turnover-gate, stairway, and the spot from turning in the B1 floor and the entire façade of space and facility expressions of these locations cannot be fully represented as it is a 3D map. 2D readers have problems with staircases. The results show that subjects using 2D maps have greater difficulty in making floor changes than 3D map users. The verbalizations of the way-finding problem for 2D map subjects are also fewer than those of 3D map subjects.

Table 4: Results of verbalization on map-reading and way-finding in Task 2

\begin{tabular}{lllll}
\hline & & 2D map & 3D map & total \\
\hline \multirow{2}{*}{ Map-reading } & & & \\
& Strategy verbalizations & & & \\
& Direction strategy & $61(51 \%)$ & $93(70 \%)$ & \\
& Floor strategy & $59(49 \%)$ & $40(30 \%)$ & \\
& Planning smoothly (n) & $113(82 \%)$ & $120(75 \%)$ & $133(69 \%)$ \\
& Problematic (n) & $13(9 \%)$ & $30(19 \%)$ & $43(22 \%)$ \\
& Memorizing route (n) & $12(9 \%)$ & $6(6 \%)$ & $18(9 \%)$ \\
& Total & $137(100 \%)$ & $160(100 \%)$ & $194(100 \%)$ \\
& & & & \\
\hline \multirow{2}{*}{ Way-finding } & recognizing route smoothly (n) & $59(50 \%)$ & $78(45 \%)$ & $137(47 \%)$ \\
& problematic (n) & $\mathbf{2 4 ( 2 0 \% )}$ & $\mathbf{4 5}(\mathbf{2 6} \%)$ & $69(24 \%)$ \\
& search (n) & $8(7 \%)$ & $9(5 \%)$ & $17(6 \%)$ \\
& recalling route (n) & $28(23 \%)$ & $39(23 \%)$ & $67(23 \%)$ \\
& Total & $119(100 \%)$ & $171(100 \%)$ & $290(100 \%)$ \\
\hline
\end{tabular}

\section{Results of route description}

The results of route description show that $60 \%$ of verbalizations relate to elements of underground space, and $40 \%$ relate to action. Most of the subjects described the routes using path and landmark information, as listed in Table 5. Subjects regarded paths as stairways, landmarks as signs, gates, exits, shops, elevators, underground shopping streets, information centers, and districts as floors, MRT line areas, and traffic systems. The quantity of verbal reporting regarding space elements and actions used by 2D map subjects exceeded that used by 3D map subjects. Since the way-finding effect of 2D map subjects is better than that of $3 \mathrm{D}$ map subjects, this study finds that the way-finding effect by using 
action for route memorization is more effective in subway stations. Notably, 2D map subjects use walls in route description. Wall surfaces are being used to memorize spatial features. On the other hand, 3D map subjects do not use wall surfaces in their route description.

Table 5: Results of route description

\begin{tabular}{lccccccrr}
\hline \multicolumn{7}{c}{ Elements of underground space } & Action & Total $^{*}$ \\
\hline & Path & Wall & Districts & Nodes & Landmarks & Total $^{*}$ \\
2D map & 11 & 5 & 8 & 0 & 12 & $36(57.1 \%)$ & $27(42.8 \%)$ & $63(100 \%)$ \\
3D map & 18 & 0 & 5 & 0 & 11 & $34(63.0 \%)$ & $20(37.0 \%)$ & $54(100 \%)$ \\
Total & 29 & 5 & 13 & 0 & 23 & $70(59.8 \%)$ & $47(40.2 \%)$ & $117(100 \%)$ \\
& & & & & & & & \\
\hline
\end{tabular}

The reasons for the mistakes made by participants are explored via route description and their verbalizations of way-finding. All of the erroneous turns occur in the B1 floor. The reasons for erroneous turns are divided into two categories: 1) failing to check landmark: Two subjects (PJ, WY) failed to locate the information centre. Three subjects (LJ, SY, and TW) failed to find the elevator. Subject CS failed to find a stairway to go upwards. Subject $\mathrm{ZH}$ failed to find the required directional sign; 2) Erroneous or inaccurate route memorization: Subject CE made the erroneous turn due to failure to remember. Subject YS was not sufficiently precise regarding his description of actions as the difference between left u-turn and right u-turn caused him to make way-finding mistakes.

Thus, generally, way-finding errors often occur when subjects expect to find a landmark in an expected location based on map-reading but then discover that the landmark is not where they expect it to be. Maps do not indicate the obstruction of wall surface and the location of directional signs, so map users can identify it by their anticipation and eventually fail in their way-finding. Therefore, differences are to be expected between the information obtained from map-reading and that obtained from the environment during way-finding, and these differences must also be considered during map design.

\section{0 Conclusion}

The results of the questionnaire survey reveal map design at Taipei Station is insufficient. In task 1 , all subjects were asked to reach an exit without reading a map and no one found the shortest way to the nearest exit. Those who were familiar with the set were shown the homing feature, and thus select the more distant but familiar exit. Those who were unfamiliar with the set could not predict whether they would arrive at the closer or more distant exit but just followed the directional signs. 
Task 2 explored the difference between the use of 2D and 3D maps via observation of subjects' behaviour, verbal reports, and route description. 2D map subjects' way-finding performance was superior to that of 3D map subjects. 2D map subjects had problems with stairway connections, but 3D map subjects were confused by the incompleteness of symbols. 2D map subjects used wall and action-based information to construct their route knowledge, but 3D map subjects tended to rely on landmarks. However, this approach made subjects vulnerable to erroneous turns and detours as a result of missing landmarks during their way-finding. Based on the investigation, the results regarding the map design of the subway station are presented as follows:

a. Support direction and floor strategies for 3-D navigation

In the 2D map, each element of space can be completely presented. In the 3D map, the connection of levels can be obviously presented. Travelers can obtain subway spatial information from both 2D and 3D maps. Subway space can be comprehended quickly and routes can be planned efficiently. So, a 3D map should be installed near a 2D map.

b. Strengthen the presentation of stairway symbols and wall surface symbols 2D map subjects were confused in stairway location and walking direction during way-finding because all stairway symbols on the map are the same. The legibility of stairway symbols on maps should be strengthened and seriously considered in map design.

c. Reduce the difference between real world and map

Way-finding tasks indicate that most erroneous decision-making results from subjects overestimating the amount of environmental information they are likely to obtain. Scale is the most important tool for distance identifying on a general map. However, scale in an indoor map is usually ignored. Maps of large subway stations are drawn according to a small scale, so the distance determination becomes difficult. Therefore, scale should also be clearly marked in a subway station map. Moreover, marking the location of important directional signs and strengthening landmarks with photos can help users remember routes and understand the space easily.

d. Labeling of the nearest exit and landmark near it

Task 1 results showed that insufficient distance information was provided by the directional sign. Maps thus can help people reduce the distance and time spent on way-finding provided the location of the nearest exit and landmark near to this exit are clearly indicated.

\section{Acknowledgement}

This research is financially supported by Ministry of Education, Taiwan and Chiba University, Japan. 


\section{References}

Chieh-Hsin Tang, Ching-Yuan Lin and Yu-Min Hsu. (2008). Exploratory research on reading cognition and escaperoute planning using building evacuation plan diagrams, Applied Ergonomics, 39 (2), March 2008, 209-217.

Christoph H Ischera, Tobias Meilingera, b, Georg Vrachliotisa, c, Martin Bro samlea, Markus Knauffa. (2006). Up the down staircase: Way-finding strategies in multi-level buildings, Journal of Environmental Psychology (2006)26 284-299.

Christoph Holscher, Simon J. Buchner, Tobias Meilinger and Gerhard Strube. (2008). Adaptivity of way-finding strategies in a multi-building ensemble: The effects of spatial structure, task requirements, and metric information, Journal of Environmental Psychology xxx 1-12.

David Brosset, Christophe Claramunt and Eric Saux. (2007). A location and action-based model for route description, Springer Berlin / Heidelberg.

Ding-Lin Chen. (2007). A Study of Guides on Human Evacuation Behaviours in MRT Underground Stations, p1011, National Taipei University of Technology, The Department of Civil Engineering, master study, Taiwan.

NIED: National Research Institute for Earth Science and Disaster Prevention. (2002).

Report of the National Research Institute for Earth Science and Disaster Prevention, Japan.

Reginald G. Golledge, Johns Hopkins. (1998). Way-finding Behaviour: Cognitive Mapping and Other Spatial Processe, Johns Hopkins Univ Pr.

Rob Kitchin. (2002). The cognition of geograthic space, Mark Blades, I. B. Tauris. Sanja Dumisevic and Sevil Sariyildiz. (2001). A systematic quality assessment of underground

spaces-public transport stations, Elsevier Science Ltd. 18(1), 13-23.

Sanja Dumisevic. (2002). Perception Aspects in Underground Space using Intelligent Knowledge Modeling, DUP Science.

Torgny Ottosson. (1987). map-reading and way-finding, Acta Universitatis Gothoburgensis, 70. 